\title{
Plasma and Muscle Free Carnitine Deficiency Due to Renal Fanconi Syndrome
}

Isa Bernardini, William B. Rizzo, Marinos Dalakas, Juan Bernar, and William A. Gahl

Section on Biochemical Genetics, Human Genetics Branch, National Institute of Child Health and Human Development, National Institutes of Health, Bethesda, Maryland 20205; Departments of Pediatrics and Human Genetics, Medical College of Virginia, Richmond, Virginia 23298; Infectious Disease Branch, National Institute of Neurological and Communicative Disorders and Stroke, National Institutes of Health, Bethesda, Maryland 20205

\section{Abstract}

Plasma and urine free and acyl carnitine were measured in 19 children with nephropathic cystinosis and renal Fanconi syndrome. Each patient exhibited a deficiency of plasma free carnitine (mean $11.7 \pm 4.0[\mathrm{SD}] \mathrm{nmol} / \mathrm{ml})$ compared with normal control values $(42.0 \pm 9.0 \mathrm{nmol} / \mathrm{ml})(P<0.001)$. Mean plasma acyl carnitine in the cystinotic subjects was normal. Four subjects with Fanconi syndrome but not cystinosis displayed the same abnormal pattern of plasma carnitine levels; controls with acidosis or a lysosomal storage disorder (Fabry disease), but not Fanconi syndrome, had entirely normal plasma carnitine levels. Two postrenal transplant subjects with cystinosis but without Fanconi syndrome also had normal plasma carnitine levels. Absolute amounts of urinary free carnitine were elevated in cystinotic individuals with Fanconi syndrome. In all 21 subjects with several different etiologies for the Fanconi syndrome, the mean fractional excretion of free carnitine (33\%) as well as acyl carnitine $(26 \%)$ greatly exceeded normal values (3 and 5\%, respectively). Total free carnitine excretion in Fanconi syndrome patients correlated with total amino acid excretion $(r=0.76)$. Two cystinotic patients fasted for $24 \mathrm{~h}$ and one idiopathic Fanconi syndrome patient fasted for $5 \mathrm{~h}$ showed normal increases in plasma $\beta$-hydroxybutyrate and acetoacetate, which suggested that hepatic fatty acid oxidation was intact despite very low plasma free carnitine levels. Muscle biopsies from two cystinotic subjects with Fanconi syndrome and plasma carnitine deficiency had 8.5 and $13.1 \mathrm{nmol}$ free carnitine per milligram of noncollagen protein, respectively (normal controls, 22.3 and 17.1); total carnitines were 11.8 and $13.3 \mathrm{nmol} / \mathrm{mg}$ noncollagen protein (controls 33.5, 20.0). One biopsy revealed a mild increase in lipid droplets. The other showed mild myopathic features with variation in muscle fiber size, small vacuoles, and an increase in lipid droplets. In renal Fanconi syndrome, failure to reabsorb free and acyl carnitine results in a secondary plasma and muscle free carnitine deficiency.

\section{Introduction}

The renal tubular Fanconi syndrome consists of a generalized transport defect in tubular reabsorption of small molecules, including water, glucose, organic acids, amino acids, and

Portions of this work were presented to the 35th Annual Meeting of the American Society of Human Genetics, Toronto, 1984.

Address correspondence and reprint requests to Dr. Gahl, Section on Biochemical Genetics.

Received for publication 16 October 1984 and in revised form 27 December 1984.

The Journal of Clinical Investigation, Inc.

Volume 75, April 1985, 1124-1130 inorganic ions such as phosphate, sodium, potassium, calcium, magnesium, and bicarbonate. Individuals with nephropathic cystinosis, who have growth retardation and eventually develop renal insufficiency, represent the largest group of children with a known etiology for their Fanconi syndrome, which usually appears by 1 yr of age (1). As a result of their Fanconi syndrome, patients with cystinosis experience the consequences of poor tubular reabsorption, including vasopressin-resistant polyuria which leads to dehydration, acidosis, and hypophosphatemic rickets. Conservative therapy is designed to correct renal losses by provision of supplemental fluids, electrolytes, alkali, phosphate, and calcium $(1,2)$.

This form of treatment, however, supplies only those nutrients generally known to be deficient in Fanconi syndrome patients. A number of other molecules normally reabsorbed by the kidney may also be lost by these patients, thus requiring replacement. One possible lost substance is carnitine, $\beta$-hydroxy$\gamma$-trimethylaminobutyric acid, a small molecule that is similar in size and charge distribution to an amino acid. Carnitine, which exists either free or as a fatty acyl ester, is required for transport of long chain fatty acids across the inner mitochondrial membrane before fatty acid oxidation and energy production $(3,4)$. Human cardiac and skeletal muscle, and to a lesser extent liver and kidney, contain relatively high amounts of carnitine, which is supplied to muscle and other noncarnitineproducing tissues by transport from the plasma (5). The plasma concentration of carnitine varies with renal filtration $(6,7)$ and reabsorption (8), potentially making plasma carnitine levels vulnerable to reduction in renal Fanconi syndrome. Carnitine depletion can be secondary or primary, and is characterized clinically by cardiomyopathy, skeletal myopathy, or fasting hypoglycemia $(3,9-14)$.

In this paper, we report plasma and urinary concentrations of free, total, and acylated carnitine in 22 subjects with the Fanconi syndrome, including 19 children with nephropathic cystinosis, a disorder of cystine transport across the lysosomal membrane $(15,16)$. Their values were compared with those of normal individuals and control subjects with related disorders. Tissue (muscle) levels of carnitine were also measured in two cystinotic children with Fanconi syndrome and plasma carnitine deficiency.

Subjects (see Table I). Children with nephropathic cystinosis age $1-11 \mathrm{yr}$ were diagnosed on the basis of typical corneal cystine crystals, renal Fanconi syndrome, and elevated leucocyte cystine content (1). Most were enrolled in the National Collaborative Cysteamine Study and received an average of 58 $\mathrm{mg} / \mathrm{kg}$ per $\mathrm{d}$ of cysteamine free base in four divided doses. Cysteamine is a free thiol that is used as a cystine-depleting agent with some clinical effectiveness $(17,18)$. Subject 18 was receiving pantethine rather than cysteamine as a cystinedepleting agent (19). Cystinotic patients also received supplemental polycitra, potassium, phosphate, and calcium as needed. 
Two brothers with oculocerebrorenal (Lowe) syndrome were diagnosed on the basis of congenital cataracts, mental retardation, renal tubular acidosis, proteinuria, and aminoaciduria. They received supplemental phosphate and citrate. One patient with an idiopathic form of Fanconi syndrome (A) had hepatomegaly and corneal clouding with electrolyte imbalance. Another (B) had hepatomegaly, severe rickets, ketonuria, and fasting hypoglycemia without lactic acidosis. Two postrenal transplant cystinosis patients had each received a kidney transplant $4 \mathrm{yr}$ before testing. The older of the two was taking prednisone every other day, plus azathioprine and L-thyroxine. The younger was receiving prednisone every other day, plus azathioprine, propranolol, and hydralazine. Although cystine continued to accumulate in all nonrenal tissues of these subjects, they had functioning kidneys with creatinine clearances of 107 and $54 \mathrm{ml} / \mathrm{min}$ per $1.73 \mathrm{~mm}$, respectively. Two brothers with 5-oxoprolinuria and deficient fibroblast glutathione synthetase activity (20) had acidosis treated with sodium citrate ( 1.7 and $3.2 \mathrm{meq} / \mathrm{kg}$ per d, respectively). A 15-yr-old boy with Fabry disease had typical corneal opacities, scrotal and umbilical angiokeratomata, acroparesthesias, and $\alpha$-galactosidase A deficiency documented in both plasma and leukocytes. His renal function, including tubular reabsorption, was unimpaired and he was receiving no medication at the time of this study.

All subjects were either inpatients or outpatients at the National Institutes of Health Clinical Center or the Medical College of Virginia. Informed consent was obtained from these patients or from their parents. Normal controls consisted of anonymous blood bank donors and volunteers.

\section{Methods}

$1-{ }^{14} \mathrm{C}$-Acetyl coenzyme A $(50 \mathrm{mCi} / \mathrm{mmol})$ was obtained from New England Nuclear (Boston, MA) and from ICN Pharmaceuticals (Irvine, $\mathrm{CA}$, and Plainview, $\mathrm{NJ}$ ), which also supplied sodium tetrathionate. Ultrapure Tris was from Boehringer-Mannheim Biochemicals (Indianapolis, IN), and AG1- $\times 8$ resin, 200-400 mesh, was from Bio-Rad Laboratories (Richmond, CA). Acetyl coenzyme A, L-carnitine and carnitine acetyltransferase were products of Sigma Chemical Co. (St. Louis, MO).

Free and total carnitine in plasma and urine were assayed according to McGarry and Foster (21), except that AG1- $\times 8$ resin was used in place of the obsolete Dowex 1- $\times 10$. Addition of 0-6 nmol of carnitine to plasma gave recovery rates of $80-90 \%$. The intraassay and interassay coefficients of variation were $2.7 \%(n=10)$ and $7.8 \%(n=5)$, respectively. Heparinized or sodium EDTA plasma, obtained from nonfasting subjects, as well as $24-\mathrm{h}$ urine aliquots, were frozen at $-20^{\circ} \mathrm{C}$ for $<3$ mo, during which time there was no reduction in measured carnitine content.

Open muscle biopsies, taken from the quadriceps muscle, were fresh-frozen in isopentane, cooled to $-160^{\circ} \mathrm{C}$ in liquid nitrogen, and processed for muscle enzyme histochemistry as described (22). Muscle carnitine was measured as described (23). Creatinine was assayed by the Jaffe reaction. Urine dipsticks for ketones indicated that none of the Fanconi syndrome patients had sufficient acetoacetate to cause an error in the creatinine or creatinine clearance determinations using this method $(24,25)$. Blood levels of acetoacetate and $\beta$-hydroxybutyrate were measured as described (26). Dicarboxylic acids were examined by gas chromatography.

Amino acids in urine, deproteinized with $5 \%$ sulfosalicylic acid, were quantitated using an LKB 4150 alpha amino acid analyzer (LKB Biochrom Ltd., Cambridge, United Kingdom) with five lithium buffers. As a measure of total amino acid output in a 24-h urine, 21 amino acids were quantitated: aspartic acid, hydroxyproline, threonine, serine, asparagine, glutamic acid, glutamine, proline, glycine, alanine, valine, one-half cystine, methionine, isoleucine, leucine, tyrosine, phenylalanine, ornithine, lysine, histidine, and arginine. Total daily amino acid excretion for nine normal subjects (four adults and five children) was 94.0 \pm 45.1 (SD) $\mu \mathrm{mol} / \mathrm{kg}$ per d (range 38.5-155.1); for 21 children with Fanconi syndrome, total amino acid excretion was $1,085.0 \pm 725.3$ (SD) $\mu \mathrm{mol} / \mathrm{kg}$ per $\mathrm{d}$ (range 342.1-3,554.5).

Fractional excretion of carnitine was calculated as $100 \times$ (urine carnitine $\times$ serum creatinine)/(serum carnitine $\times$ urine creatinine).

\section{Results}

Each of 19 children age 20 mo-11 yr, with nephropathic cystinosis and renal Fanconi syndrome, had a plasma free carnitine level below the normal range (Table I). As a group, children with cystinosis averaged $12 \mathrm{nmol}$ free carnitine per milliliter of plasma, which was significantly less than the normal mean of $42 \mathrm{nmol} / \mathrm{ml}(P<0.001)$. Plasma acylcarnitine levels were not decreased, so the large difference in total plasma carnitine between cystinotic and normal subjects $(P$ $<0.001$ ) reflected the substantial free carnitine deficiency in the patients. The abnormally low free carnitine levels in cystinotic children were not related to age, serum creatinine, or cysteamine therapy. (Subjects 1, 12, 16, and 18 were not receiving cysteamine.) Subject 15 , with late onset cystinosis and mild aminoaciduria, had a plasma free carnitine level approaching the normal range.

Two brothers with oculocerebrorenal syndrome and Fanconi syndrome had low free plasma carnitine levels (Table I). Two other patients with severe idiopathic forms of Fanconi syndrome also had markedly decreased free and total plasma carnitines. In contrast, two brothers with 5-oxoprolinuria and acidosis, but not Fanconi syndrome, had normal free, acyl, and total plasma carnitines. A boy with the lysosomal storage disorder, Fabry disease, but without renal dysfunction, also had plasma carnitine levels in the normal range. Finally, two postrenal transplant subjects who had cystinosis but not Fanconi syndrome had normal plasma carnitine levels and normal or nearly normal fractional carnitine excretions (Table I).

As others have reported (27-30), daily urinary excretion of free and acylcarnitine varied widely in all subjects, including the cystinotic patients (Table I). Free carnitine excretion in cystinotics $(4.8 \pm 1.8 \mu \mathrm{mol} / \mathrm{kg}$ per $\mathrm{d})$ exceeded that in normal subjects $(3.0 \pm 0.5 \mu \mathrm{mol} / \mathrm{kg}$ per $\mathrm{d})$, while acyl carnitine excretion did not differ significantly from normal (Table I) (26). Nevertheless, the amounts of urinary free carnitine are abnormal in the presence of very low plasma levels. In fact, the mean fractional excretions of free and acyl carnitine in 18 cystinotic patients, two oculocerebrorenal syndrome patients with Fanconi syndrome, and one idiopathic Fanconi syndrome subject were $33.2 \pm 28.3$ (SD) $\%$ and $25.8 \pm 17.1 \%$, respectively. These values far exceeded previously published norms $(6,8,28)$ for free carnitine fractional excretion $(1-2 \%)$, as well as our own control values for both free $(2.8 \pm 0.9 \%)$ and acyl $(5.3 \pm 2.2 \%)$ carnitine fractional excretions (Table I, $P<0.001$ ).

To further investigate the relationship between carnitine excretion and renal Fanconi syndrome, total daily urinary free carnitine was plotted against total daily amino acid excretion (see Methods) for the 21 subjects with Fanconi syndrome and amino aciduria (Fig. 1). Free carnitine excretion increased directly with amino acid excretion $(r=0.76)$. Subject 15 was unusual in that she had late onset cystinosis that was diagnosed at $6 \mathrm{yr}$ of age, mild amino aciduria, mild carnitine deficiency (Table I), and was a carrier for $\alpha$-galactosidase A deficiency. 
Table I. Plasma and Urine Carnitine in Patients with Cystinosis and Other Disorders

\begin{tabular}{|c|c|c|c|c|c|c|c|c|c|c|}
\hline \multirow[b]{3}{*}{ Subjects } & \multirow[b]{3}{*}{ Age } & \multirow[b]{3}{*}{ Serum $\mathrm{Cr}$} & \multicolumn{8}{|l|}{ Carnitine } \\
\hline & & & \multicolumn{3}{|c|}{ Plasma $(n m o l / m l)$} & \multicolumn{3}{|c|}{ Urine $(\mu \mathrm{mol} / \mathrm{kg} / \mathrm{d})$} & \multicolumn{2}{|c|}{ Fractional excretion (\%) } \\
\hline & & & Free & Acyl & Total & Free & Acyl & Total & Free & Acyl \\
\hline & $y r-m o$ & $m g / d l$ & & & & & & & & \\
\hline \multicolumn{11}{|l|}{ Normal controls* } \\
\hline $\mathbf{N}$ & & & 15 & 15 & 15 & 6 & 6 & 6 & 6 & 6 \\
\hline Mean & & & 42.0 & 10.3 & 52.3 & 3.0 & 3.3 & 6.3 & 2.8 & 5.3 \\
\hline SD & & & 9.0 & 4.1 & 11.4 & 0.5 & 0.6 & 0.8 & 0.9 & 2.2 \\
\hline Range & & & $26-66$ & $3-18$ & $33-84$ & $2.4-3.7$ & $2.4-3.9$ & $4.9-7.1$ & $1.6-4.0$ & $2.3-9.0$ \\
\hline \multicolumn{11}{|l|}{ Cystinoticsł } \\
\hline 1 & $1-8$ & 1.0 & 9 & 32 & 41 & 7.2 & 2.7 & 9.8 & 35 & 5 \\
\hline 2 & $2-2$ & 0.4 & 10 & 1 & 11 & 3.0 & 3.5 & 6.5 & 7 & 15 \\
\hline 3 & $3-2$ & 2.8 & 10 & 13 & 23 & 3.2 & - & - & 99 & - \\
\hline 4 & $3-11$ & 0.6 & 16 & 5 & 21 & 4.0 & 4.8 & 8.9 & 8 & 36 \\
\hline 5 & $4-9$ & 1.0 & 9 & 2 & 11 & 1.6 & 0.8 & 2.3 & 10 & 22 \\
\hline 6 & $5-0$ & 0.6 & 12 & 6 & 18 & 8.6 & 5.1 & 13.7 & 14 & 16 \\
\hline 7 & $5-0$ & 0.7 & 11 & 3 & 14 & 4.7 & 5.2 & 9.9 & 11 & 46 \\
\hline 8 & $5-6$ & 0.6 & 11 & 8 & 19 & 4.3 & 1.6 & 5.9 & 9 & 5 \\
\hline 9 & $5-8$ & 1.7 & 8 & 11 & 19 & - & - & - & - & - \\
\hline 10 & $6-2$ & 0.8 & 9 & 9 & 18 & 7.0 & 4.0 & 11.0 & 27 & 17 \\
\hline 11 & $7-8$ & 1.4 & 11 & 10 & 21 & 6.7 & 1.4 & 8.1 & 40 & 5 \\
\hline 12 & $7-8$ & 1.4 & 15 & 24 & 39 & 4.2 & 6.1 & 10.3 & 21 & 19 \\
\hline 13 & $8-2$ & 0.8 & 11 & 4 & 15 & 3.3 & 6.7 & 10.0 & 8 & 45 \\
\hline 14 & $8-6$ & 1.4 & 7 & 9 & 16 & 3.4 & 2.2 & 5.7 & 30 & 19 \\
\hline 15 & $8-8$ & 1.0 & 25 & 9 & 34 & 6.8 & 5.7 & 12.5 & 14 & 27 \\
\hline 16 & $8-11$ & 6.0 & 14 & 21 & 35 & 3.6 & 1.7 & 5.3 & 85 & 34 \\
\hline 17 & $9-7$ & 2.6 & 12 & 7 & 19 & 5.6 & 5.6 & 11.2 & 44 & 75 \\
\hline 18 & $10-0$ & 2.5 & 13 & 16 & 29 & 4.4 & 3.9 & 8.3 & 49 & 32 \\
\hline 19 & $11-3$ & 2.9 & 10 & 6 & 16 & 4.6 & 2.4 & 7.0 & 70 & 35 \\
\hline Mean & & & 11.7 & 10.3 & 22.1 & 4.8 & 3.7 & 8.6 & 32 & 27 \\
\hline SD & & & 4.0 & 8.0 & 9.1 & 1.8 & 1.9 & 2.9 & 28 & 18 \\
\hline \multicolumn{11}{|l|}{$P$ (compared with } \\
\hline controls) & & & $<0.001$ & NS & $<0.001$ & $<0.005$ & NS & $<0.01$ & $<0.001$ & $<0.001$ \\
\hline \multicolumn{11}{|l|}{ Others } \\
\hline \multicolumn{11}{|l|}{ Oculocerebrorenal } \\
\hline \multicolumn{11}{|l|}{ Oculocerebrorenal } \\
\hline (Fanconi) syndrome & $11-6$ & 1.0 & 18 & 19 & 37 & 2.5 & 3.3 & 5.7 & 8 & 15 \\
\hline \multicolumn{11}{|l|}{ Idiopathic Fanconi } \\
\hline \multicolumn{11}{|l|}{ Idiopathic Fanconi } \\
\hline syndrome (B) & $1-1$ & 0.5 & 10 & 8 & 18 & - & - & - & - & - \\
\hline \multicolumn{6}{|l|}{ Cystinotic post-renal } & 3.1 & & & 3 & 6 \\
\hline \multicolumn{11}{|l|}{ Cystinotic post-renal } \\
\hline transplant & $16-3$ & 1.0 & 47 & 10 & 57 & 7.2 & 1.4 & 8.6 & 7 & 6 \\
\hline 5-oxoprolinuria & $6-2$ & 0.7 & 48 & 7 & 55 & - & - & - & - & - \\
\hline 5-oxoprolinuria & $8-9$ & 0.6 & 47 & 9 & 56 & - & - & - & - & - \\
\hline Fabry disease & $15-1$ & 0.8 & 48 & 4 & 52 & 6.8 & 1.1 & 7.9 & 5 & 9 \\
\hline
\end{tabular}

* All adults except three children, age 4-7 yr, whose values did not differ significantly from those of adults. Data are similar to those published for adults $(3,12)$ and children $(28,29)$. ‡ Cystinotic patients were receiving $43-74 \mathrm{mg} / \mathrm{kg}$ per d of cysteamine free base, divided every $6 \mathrm{~h}$, except: case 1, newly diagnosed, not yet on cysteamine; case 12, off cysteamine for religious reasons; case 16, off cysteamine due to renal failure; case 18, receiving pantethine (19) instead of cysteamine. Case 15 had late-onset cystinosis. 


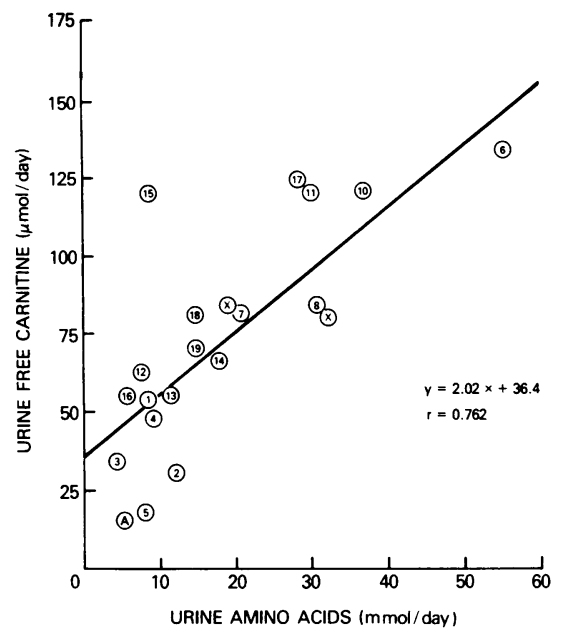

Figure 1. Relationship between urinary free carnitine and amino acid excretion in subjects with renal Fanconi syndrome. Each number represents the corresponding cystinotic subject listed in Table I. The symbols $(x)$ refer to the brothers with oculocerebrorenal syndrome, and (A) refers to one of the idiopathic Fanconi syndrome subjects. Amino acid excretion is defined in Methods.

Excluding her data, the correlation coefficient for the data in Fig. 1 was 0.86 . Free carnitine excretion correlated poorly with weight $(r=0.54)$, and there was little correlation between acylcarnitine excretion and amino acid excretion $(r=0.30)$.

Since most of the Fanconi syndrome patients were deficient in plasma free carnitine to the same extent as reported in systemic carnitine deficiency (6), we examined whether they were functionally impaired in fatty acid oxidation as well. Three patients underwent a fasting study (Table II). Two cystinotic patients showed no evidence of hypoglycemia or dicarboxylic aciduria after $24 \mathrm{~h}$ of fasting; they also had a normal rise in $\beta$-hydroxybutyrate and acetoacetate levels (31). The patient with idiopathic Fanconi syndrome became hypoglycemic after $5 \mathrm{~h}$ of fasting, but also showed a normal rise in plasma $\beta$-hydroxybutyrate and acetoacetate. Assuming constant ketone utilization, this would indicate increased ketone production consistent with normal hepatic fatty acid oxidation.
Two patients with Fanconi syndrome and carnitine deficiency underwent quadriceps muscle biopsies. Subject 11 had muscle free and total carnitine levels of 13.1 and $13.3 \mathrm{nmol} /$ $\mathrm{mg}$ of noncollagen protein; the values for subject 5 were 8.5 and 11.8. Our normal control values of 17.1 and $22.3 \mathrm{nmol}$ of free carnitine and 20.0 and $33.5 \mathrm{nmol}$ of total carnitine per milligram of noncollagen protein correspond well to literature values $(3,11)$. The biopsy from subject 11 showed increased lipid droplets. The muscle of subject 5 revealed mild myopathic features with variation in muscle fiber size, small vacuoles, occasional ragged-red fibers, and increased accumulation of lipid droplets with Sudan black and oil-red-O stains (Fig. 2). These morphologic findings are consistent with muscle carnitine deficiency.

\section{Discussion}

In man, the biosynthesis of carnitine begins with lysine (4). The conversion of lysine to butyrobetaine occurs in most tissues, but hydroxylation of butyrobetaine to carnitine takes place largely in the liver, kidney, and testis (5). Carnitine is transported through the blood to tissues such as skeletal and cardiac muscle, which require it for transport of long chain fatty acids into mitochondria. The uptake of carnitine by different organs is determined by tissue-specific fluxes from the plasma $(5,32)$. Plasma concentrations are influenced by diet as well as de novo synthesis, and deficiency states due to poor dietary intake in neonates (33), as well as apparently impaired production of carnitine (13), have been reported.

Recently, however, the role of the kidney in regulating plasma carnitine levels has been increasingly recognized. Carnitine is filtered by the kidney; hence, there are elevated muscle (34) and plasma (35) levels of carnitine in patients with renal insufficiency. Hemodialysis lowers free carnitine levels $(34,35)$. This finding, as well as in vitro equilibrium dialysis experiments (36), indicates that carnitine is unbound or only loosely bound by plasma proteins. Thus, the maintenance of normal plasma carnitine levels depends on tubular reabsorption, which under normal circumstances efficiently reclaims $98-99 \%$ of the filtered load of free carnitine $(6,8,28)$ and $77-99 \%$ of the filtered load of acyl carnitine (28). The

Table II. Effect of Fasting on Fatty Acid Oxidation in Carnitine-deficient Fanconi Syndrome Subjects

\begin{tabular}{|c|c|c|c|c|c|c|}
\hline \multirow[b]{2}{*}{ Subject } & \multirow[b]{2}{*}{ Period of fasting } & \multicolumn{2}{|c|}{$\begin{array}{l}\text { Plasma carnitine } \\
(\mathrm{nmol} / \mathrm{ml})\end{array}$} & \multirow[b]{2}{*}{ Glucose } & \multirow[b]{2}{*}{$\beta$-Hydroxybutyrate } & \multirow[b]{2}{*}{ Acetoacetate } \\
\hline & & Free & Acyl & & & \\
\hline & $h$ & & & $m g / d l$ & $m M$ & $m M$ \\
\hline \multirow[t]{2}{*}{ Cystinotic 7} & 0 & 11 & 20 & 113 & 0.23 & 0.02 \\
\hline & 24 & 8 & 34 & 58 & 2.07 & 0.19 \\
\hline \multirow{2}{*}{ Cystinotic 13} & 0 & 7 & 17 & 119 & 0.36 & 0.04 \\
\hline & 24 & 8 & 32 & 66 & 2.46 & 0.35 \\
\hline Idiopathic Fanconi syndrome & 0 & 10 & 8 & 125 & 0.28 & 0.12 \\
\hline (B) & 5 & 5 & 9 & 21 & 1.36 & 0.71 \\
\hline \multirow[t]{2}{*}{ Normals* } & 0 & - & - & $83-116$ & $0.13-0.37$ & $0.01-0.04$ \\
\hline & 24 & - & - & $35-67$ & $0.79-4.12$ & $0.03-0.42$ \\
\hline
\end{tabular}

\footnotetext{
* Combined range of reported normal children (31) and five children studied at the Medical College of Virginia (Richmond, VA).
} 


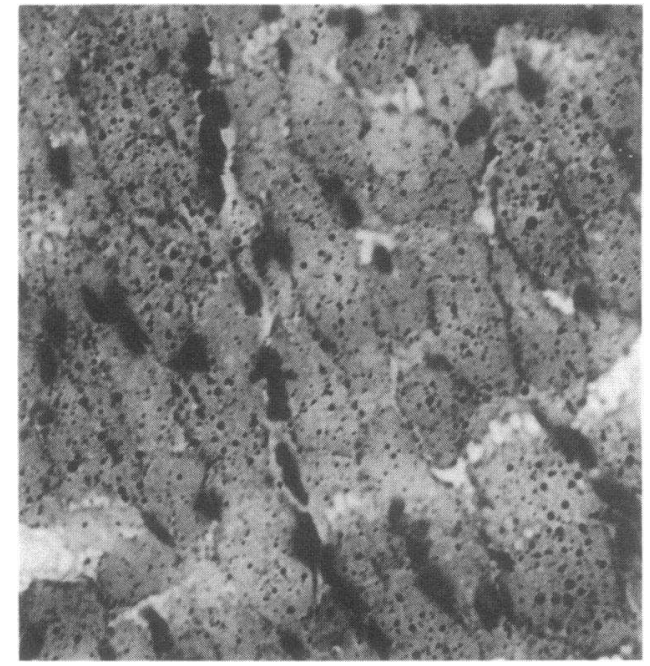

Figure 2. Transverse frozen section of muscle biopsy from subject 5 , stained with oil-red-O, showing increased number of lipid droplets. $\times 350$.

transport of L-carnitine across rat renal brush border membranes has been reported to be structure specific and sodium gradient dependent (37). It has not been determined whether carnitine is acylated in the kidney before excretion, or whether any form of carnitine is secreted. However, carnitine infusions have resulted in an increased fractional excretion of both free and total carnitine, which is consistent with a discrete tubular reabsorption maximum for both free and acyl carnitine (6). Although this was not specifically measured in the present study, patients with cystinosis may have a markedly reduced tubular reabsorption maximum for carnitine as part of their Fanconi syndrome (Table I, Fig. 1); the role of reabsorption in the renal handling of free carnitine is emphasized by the fact that even cystinotic children with increased serum creatinines manifest very low plasma free carnitine levels (Table I).

All 21 individuals with Fanconi syndrome had increased fractional excretions of both free and acyl carnitine without carnitine loading. This abnormality was manifest by a reduction in mean plasma free carnitine concentration to $28 \%$ of normal (Table I). The normalcy of plasma acyl carnitine may be related to the relative rates of synthesis (interconversion with free carnitine) and catabolism which, along with excretion, determine steady state levels.

The high fractional excretions and low plasma concentrations of free carnitine were related to Fanconi syndrome and not to cystinosis per se. Two cystinotic subjects who had received renal transplants and no longer had Fanconi syndrome exhibited normal plasma carnitines and fractional excretions of carnitine within or near the normal range (Table I). In addition, marked plasma carnitine deficiency was seen in four noncystinotic subjects, two with an idiopathic type of Fanconi syndrome and two with the tubular reabsorption defect of oculocerebrorenal (Lowe) syndrome. The subjects with these disorders, as well as the four cystinotic patients who were not receiving cysteamine (17), also provided evidence against the possibility that the drug cysteamine lowered free plasma carnitine levels. Normal carnitine concentrations in patients with 5-oxoprolinuria and Fabry disease indicate that the low levels in cystinotic patients were not due to generalized metabolic acidosis or lysosomal storage, respectively. The correlation between free carnitine excretion and amino acid excretion (Fig. 1) further supports the renal tubular etiology of the plasma free carnitine deficiency. The fact that free carnitine excretion correlated with amino acid excretion better than did acyl carnitine excretion helps differentiate this plasma carnitine deficiency from that found in organic acidurias $(3,38,39)$, in which acylcarnitine excretion may be the primary cause of plasma carnitine deficiency. However, this finding has been disputed (40).

Low plasma total carnitine in association with Fanconi syndrome has been reported in two individuals $(28,41-43)$, both of whom had multisystem involvement. One patient, a 3 -yr-old girl with mitochondrial cytopathy and Fanconi syndrome (28), had a fractional excretion of free carnitine of $8.2 \%$. The other patient presented at age four with psychomotor retardation, impaired liver function, hyperammonemia, an episode of hypoglycemia, muscle weakness, and renal Fanconi syndrome (41). Urinary free carnitine excretion was increased, and serum free and total carnitine were 3.5 and $9.6 \mathrm{nmol} / \mathrm{ml}$, respectively. These increased to 10.7 and $24.7 \mathrm{nmol} / \mathrm{ml}$ after 5 mo of L-carnitine treatment, along with improved muscle strength and scalp hair growth (43). Although carnitine clearances were not reported, and several organ systems were involved, this patient's carnitine deficiency was considered secondary to the Fanconi syndrome.

The plasma concentrations of free and total carnitine in our Fanconi syndrome patients were in the same range as those reported for individuals with systemic carnitine deficiency (6). However, the Fanconi syndrome patients did not appear to have hepatic carnitine deficiency. Three patients with Fanconi syndrome and plasma free carnitine deficiency who underwent a fasting study showed no deficiency in ketone body production (Table II). This would suggest that hepatic carnitine levels were not depleted enough to functionally impair fatty acid oxidation. Only the patient with idiopathic Fanconi syndrome developed hypoglycemia, but it was probably not due to his carnitine deficiency, since he had a brisk ketone body response.

In contrast to the apparent lack of hepatic involvement, patients with Fanconi syndrome do appear to manifest muscle carnitine deficiency, with moderate depressions in both free and total carnitine levels, lipid droplet accumulation (Fig. 2), and myopathic changes (subject 5). This situation is not surprising, since the muscle depends upon delivery of carnitine from the plasma for its supply, and plasma free carnitine levels are low. However, the ability to transport carnitine into muscle is not expected to be impaired in Fanconi syndrome; this differentiates the condition from primary muscle carnitine deficiency $(3,10)$ and suggests that elevating plasma free carnitine will result in repletion of muscle carnitine. The lack of hepatic involvement in Fanconi syndrome patients might also be anticipated, since the liver is provided carnitine both by its own synthetic machinery and by the portal circulation before carnitine can be spilled through the kidney.

Our subjects with cystinosis and the Fanconi syndrome do manifest poor muscular development, but no other overt signs of systemic carnitine deficiency, which is characterized by episodes of hypoglycemia, Reye syndrome-like encephalopathy, and muscle weakness $(3,10,11)$. Although patients with systemic carnitine deficiency have exhibited a renal carnitine leak, this alone cannot account for tissue carnitine depletion in the disorder, since one normal control exhibited a similar 
renal carnitine leak (6). It appears that the renal defect is only one component of a multisystem disorder, in which other organ system involvement causes the more severe clinical manifestations of systemic carnitine deficiency. Because only one other organ system (muscle) appears secondarily involved in Fanconi syndrome subjects, one might expect that the only clinical impairment would be poor muscular development. Nevertheless, acute stress or chronic illness may trigger other clinical manifestations in such patients.

This investigation clearly establishes plasma and muscle free carnitine deficiency as components of the various forms of renal Fanconi syndrome, and verifies that the carnitine deficiency is due to impaired tubular absorption per se, and not solely due to involvement of other organ systems.

\section{Acknowledgments}

The authors thank Dr. George Hoganson and Dr. Russell Chesney of the University of Wisconsin at Madison for their insightful comments. The technical assistance of Ms. Pat Spencer is greatly appreciated.

\section{References}

1. Schneider, J. A., and J. D. Schulman. 1983. Cystinosis. In The Metabolic Basis of Inherited Disease. J. B. Stanbury, J. B. Wyngaarden, D. S. Fredrickson, J. L. Goldstein, and M. S. Brown, editors. McGrawHill, Inc., New York. Fifth ed. 1844-1866.

2. Schulman, J. D. 1973. Cystinosis. DHEW Publication Number (NIH) 72-249, U. S. Government Printing Office, Washington, D.C. $1-258$.

3. Rebouche, C. J., and A. G. Engel. 1983. Carnitine metabolism and deficiency syndromes. Mayo Clin. Proc. 58:533-540.

4. Broquist, H. P. 1982. Carnitine biosynthesis and function. Fed. Proc. 41:2840-2842.

5. Bohmer, T., and P. Molstad. 1980. Carnitine transport across the plasma membrane. In Carnitine Biosynthesis, Metabolism and Functions. R. A. Frenkel and J. D. McGarry, editors. Academic Press, Inc., New York. 73-89.

6. Engel, A. G., C. J. Rebouche, D. M. Wilson, A. M. Glasgow, C. A. Romshe, and R. P. Cruse. 1981. Primary systemic carnitine deficiency. II. Renal handling of carnitine. Neurology. 31:819-825.

7. Bartel, L. L., J. L. Hussey, and E. Shrago. 1981. Perturbation of serum carnitine levels in human adults by chronic renal disease and dialysis therapy. Am. J. Clin. Nutr. 34:1314-1320.

8. Frohlich, J., D. W. Seccombe, P. Hahn, P. Dodek, and I. Hynie. 1978. Effect of fasting on free and esterified carnitine levels in human serum and urine: correlation with serum levels of free fatty acids and $\beta$-hydroxybutyrate. Metab. Clin. Exp. 27:555-561.

9. Engel, A. G., and C. Angelini. 1973. Carnitine deficiency of human skeletal muscle with associated lipid storage myopathy: a new syndrome. Science (Wash. DC). 179:899-902.

10. Engel, A. G. 1980. Possible causes and effects of carnitine deficiency in man. In Carnitine Biosynthesis, Metabolism and Functions. R. A. Frenkel and J. D. McGarry, editors. Academic Press, Inc., New York. 271-285.

11. Rebouche, C. J., and A. G. Engel. 1981. Primary systemic carnitine deficiency. I. Carnitine biosynthesis. Neurology. 31:813-818.

12. Ware, A. J., W. C. Burton, J. D. McGarry, J. F. Marks, and A. G. Weinberg. 1978. Systemic carnitine deficiency. Report of a fatal case with multisystemic manifestations. J. Pediatr. 93:959-964.

13. Tripp, M. E., M. L. Katcher, H. A. Peters, E. F. Gilbert, S. Ayra, R. J. Hodach, and A. L. Shug. 1981. Systemic carnitine deficiency presenting as familial endocardial fibroelastosis. A treatable cardiomyopathy. N. Engl. J. Med. 305:385-390.
14. Waber, L. J., D. Valle, C. Neill, S. DiMauro, and A. Shug. 1982. Carnitine deficiency presenting as familial cardiomyopathy: a treatable defect in carnitine transport. J. Pediatr. 101:700-705.

15. Gahl, W. A., N. Bashan, F. Tietze, I. Bernardini, and J. D. Schulman. 1982. Cystine transport is defective in isolated leukocyte lysosomes from patients with cystinosis. Science (Wash. DC). 217: 1263-1265.

16. Gahl, W. A., F. Tietze, N. Bashan, I. Bernardini, D. Raiford, and J. D. Schulman. 1982. Characteristics of cystine counter-transport in normal and cystinotic lysosome-rich leucocyte granular fractions. Biochem. J. 216:393-400.

17. Thoene, J. G., R. G. Oshima, J. C. Crawhall, D. L. Olson, and J. A. Schneider. 1976. Intracellular cystine depletion by aminothiols in vitro and in vivo. J. Clin. Invest. 58:180-189.

18. Thoene, J. G., W. A. Gahl, W. Rizzo, J. D. Schulman, G. Reed, D. Denman, J. Schlesselman, A. Jonas, and J. A. Schneider. 1983. Cysteamine therapy of nephropathic cystinosis: evidence for effectiveness. Pediatr. Res. 17:220A. (Abstr.)

19. Butler, J. D., and M. Zatz. 1983. Pantethine depletes cystinotic fibroblasts of cystine. J. Pediatr. 102:796-798.

20. Spielberg, S. P., L. I. Kramer, S. I. Goodman, J. Butler, F. Tietze, P. Quinn, and J. D. Schulman. 1977. 5-Oxoprolinuria: biochemical observations and case report. J. Pediatr. 91:237-241.

21. McGarry, J. D., and D. W. Foster. 1976. An improved and simplified radioisotopic assay for the determination of free and esterified carnitine. J. Lipid Res. 17:277-281.

22. Engel, W. K. 1970. Selective and nonselective susceptibility of muscle fiber types: a new approach to human neuromuscular diseases. Arch. Neurol. 22:97-117.

23. Cederblad, G., S. Lindstedt, and K. Lundholm. 1974. Concentration of carnitine in human muscle tissue. Clin. Chim. Acta. 53:311321.

24. Watkins, P. J. 1967. The effect of ketone bodies on the determination of creatinine. Clin. Chim. Acta. 18:191-196.

25. Blank, D. W., and A. A. Nanji. 1982. Ketone interference in estimation of urinary creatinine; effect on creatinine clearance in diabetic ketoacidosis. Clin. Biochem. 15:279-280.

26. Olsen, C. 1971. An enzymatic fluorometric micromethod for the determination of acetoacetate, $\beta$-hydroxybutyrate, pyruvate and lactate. Clin. Chim. Acta. 33:293-300.

27. Cederblad, G., and S. Lindstedt. 1971. Excretion of L-carnitine in man. Clin. Chim. Acta. 33:117-123.

28. Ohtani, Y., S. Nishiyama, and I. Matsuda. 1984. Renal handling of free and acylcarnitine in secondary carnitine deficiency. Neurology. 34:977-979.

29. Battistella, P. A., L. Vergani, F. Donzelli, F. F. Rubaltelli, and C. Angelini. 1980. Plasma and urine carnitine levels during development. Pediatr. Res. 14:1379-1381.

30. Carrier, H. N., and G. Berthillier. 1980. Carnitine levels in normal children and adults in patients with diseased muscle. Muscle \& Nerve. 3:326-334.

31. Stanley, C. A., and L. Baker. 1976. Hyperinsulinism in infancy: diagnosis by demonstration of abnormal response to fasting hypoglycemia. Pediatrics. 57:702-711.

32. Rebouche, C. J., and A. G. Engel. 1984. Kinetic compartmental analysis of carnitine metabolism in the human carnitine deficiency syndromes. Evidence for alterations in tissue carnitine transport. $J$. Clin. Invest. 73:857-867.

33. Schmidt-Sommerfeld, E., D. Penn, and H. Wolf. 1983. Carnitine deficiency in premature infants receiving total parenteral nutrition: effect of L-carnitine supplementation. J. Pediatr. 102:931-935.

34. Bertoli, M., P. A. Battistella, L. Vergani, A. Naso, M. L. Gasparotto, G. Romagnoli, and C. Angelini. 1981. Carnitine deficiency induced during hemodialysis and hyperlipidemia: effect of replacement therapy. Am. J. Clin. Nutr. 34:1496-1500.

35. Gusmano, R., and R. Oleggini. 1981. Plasma carnitine concentrations and dyslipidemia in children on maintenance hemodialysis. $J$. Pediatr. 99:429-432. 
36. Bohmer, T., H. Bergrem, and K. Eiklid. 1978. Carnitine deficiency induced during intermittent haemodialysis for renal failure. Lancet. I:126-128.

37. Rebouche, C. J., and D. L. Mack. 1984. $\mathrm{Na}^{+}-$Gradientstimulated transport of L-carnitine by rat renal brush border membrane. Fed. Proc. 42:1053/4462. (Abstr.)

38. Roe, C. R., D. S. Millington, D. A. Maltby, T. P. Bohan, and C. L. Hoppel. 1984. L-Carnitine enhances excretion of propionyl coenzyme A as propionyl carnitine in propionic acidemia. J. Clin. Invest. 73:1785-1788.

39. Engel, A. G., and C. J. Rebouche. 1984. Carnitine metabolism and inborn errors. J. Inherited Metab. Dis. 7(Suppl. 1):38-43.
40. Stanley, C. A., G. T. Berry, M. Yudkoff, R. I. Kelley, and S. Segal. 1984. Urine carnitine excretion in secondary carnitine deficiency. Pediatr. Res. 18:300A. (Abstr.)

41. Netzloff, M. L., A. F. Kohrman, M. Z. Jones, R. K. Emaus, L. L. Bieber, and S. DiMauro. 1981. Carnitine deficiency associated with renal Fanconi syndrome. J. Neuropathol. Exp. Neurol. 40:351. (Abstr.)

42. Bieber, L. L., R. Emaus, K. Valkner, and S. Farrell. 1982. Possible functions of short-chain and medium-chain carnitine acyltransferases. Fed. Proc. 41:2858-2862.

43. Netzloff, M. L., and L. L. Bieber. 1984. L-Carnitine supplementation in systemic carnitine deficiency. Pediatr. Res. 18:297A. (Abstr.) 